\title{
Coeducar las Relaciones Afectivosexuales para Promover la Igualdad Sexual y de Género y la Justicia Social
}

\author{
Coeducating Sexuality and Love Relationships to Promote Sex \\ and Gender Equality and Social Justice
}

\author{
Mar Venegas * \\ Universidad de Granada
}

\begin{abstract}
La educación, de manera destacada la formal que ocurre en la escuela, es uno de los principales motores de cambio de la sociedad. De ahí que, quienes comprometemos nuestro trabajo con la promoción del cambio social hacia la igualdad sexual y de género, localicemos en la educación (sobre todo la escolar) esta reivindicación. En línea con ello, en este artículo se empieza analizando el recorrido histórico desde la escuela segregadora, pasando por la escuela mixta, hasta llegar a la coeducación como modelo escolar. A continuación, se pone de manifiesto cómo la LOMCE favorece la segregación escolar en función del sexo. Seguidamente, se evidencian los aspectos sociales, afectivosexuales y emocionales del debate entre los tres modelos de educación antes mencionados, para situar en ellos la necesidad de apostar por la coeducación afectivosexual como alternativa a la segregación escolar por sexo. Para ello, se analizan datos secundarios procedentes de los tres únicos estudios del Centro de Investigaciones Sociológicas disponibles en España en relación a esta temática. El artículo termina con algunas conclusiones destacadas a partir del análisis de estos datos para ilustrar la necesidad de apostar por una educación feminista, a través de la coeducación afectivosexual, para promover la justicia social, propia de una sociedad democrática, mediante la promoción de la igualdad sexual y de género desde el ámbito de la educación.
\end{abstract}

Descriptores: Educación sexual, Igualdad de oportunidades, Coeducación, Movimiento de liberación femenina, Justicia social

Education, notably the formal one that happens in the school, is one of the main engines of change of the society. Hence, those who commit our work with the promotion of social change towards sexual and gender equality, let us locate this claim on education (especially the scholar one). In line with this, this article begins by analysing the historical journey from the segregating single-sex school, through the mixed school, to coeducation as a school model. It then discusses how LOMCE favours school single-sex segregation. The social, affective and sexual, and emotional aspects of the debate between the three models of education mentioned above are evidenced in order to place in them the need to bet on sex and relationships coeducation as an alternative to school segregation by sex. To do this, we analyse secondary data from the three only studies of the Centre for Sociological Research available in Spain in relation to this subject. The article concludes with some outstanding conclusions from the analysis of these data to illustrate the need to bet on a feminist education, through sex and relationships coeducation, to promote social justice, inherent of a democratic society, through the promotion of sexual and gender equality from the field of education.

Keywords: Sex education, Equal opportunity, Coeducation, Women liberation movement, Social justice.

*Contacto: mariter@ugr.es

ISSN: 2254-3139

www.rinace.net/riejs/

revistas.uam.es/riejs
Recibido: $\quad 13$ de abril 2017

$1^{\text {a }}$ Evaluación: 31 de julio 2017

$2^{\text {a }}$ Evaluación: 29 de agosto 2017

Aceptado: $\quad 15$ de septiembre 2017 
Como este monográfico sobre Educación Feminista y Justicia Social quiere platear, son muchos los estudios, pero también las muestras de vida cotidiana, que confirman la pervivencia del sexismo y los estereotipos de género en nuestra sociedad. Simultáneamente, encontramos múltiples ejemplos de cómo la lucha feminista se mantiene alerta, precisamente por la necesidad de seguir combatiéndolos. En el ámbito del análisis sociológico, suele decirse que el siglo XIX fue el siglo del movimiento obrero, y el siglo Xx el del movimiento feminista. Por suerte, son muchos los fenómenos que dan cuenta de los importantes cambios sociales que han acontecido, sobre todo, en la segunda mitad del siglo Xx, gracias a la lucha feminista. Pero también queda todavía mucho por hacer. Como bien se ha indicado en la literatura especializada al respecto, desde una perspectiva educativa, la escuela mixta ha resultado insuficiente. Sólo la escuela coeducativa puede contribuir a sentar las bases de una sociedad construida sobre la base de relaciones igualitarias entre las personas, desde el reconocimiento de la diversidad sexual y de género, y la defensa de la justicia social.

Con estas consideraciones de fondo, este artículo pretende contribuir al debate poniendo el foco en el análisis de un aspecto que desde estas páginas se entiende como fundamental precisamente para construir esas relaciones en igualdad: la educación afectivosexual en el marco del debate entre la escuela segregadora y la coeducativa. En concreto, en una primera parte del artículo se analiza este debate en síntesis, para señalar el aspecto central del debate que nos conduce a la necesidad de apostar por la educación de las relaciones afectivosexuales en igualdad. A continuación, se hace una breve mención a las políticas de igualdad en educación para ilustrar este análisis e introducir la relación entre coeducación y educación afectivosexual. Seguidamente, se analiza el fenómeno en cifras, a través de un análisis secundario de los pocos datos sociológicos ofrecidos en España por los estudios del Centro de Investigaciones Sociológicas (2008a, 2008b, 2010). El artículo concluye con algunas consideraciones que pretenden ilustrar el objetivo con el que parte: la necesidad de apostar por la coeducación de las relaciones afectivosexuales para promover la igualdad y la justicia social.

\section{El sistema educativo en perspectiva histórica: el debate entre escuela segregadora, escuela mixta y escuela coeducadora}

En España (Blat, 1994; Fernández-González y González, 2015; Rodríguez-Martínez, 2011; Subirats, 1994, 2010, 2013; Subirats y Brullet, 2002; Venegas, 2015), como en otros países (Araújo, 2000; Arnot y Weiler, 1993; Duru-Bellat, 1990), han proliferado los estudios que, a partir de los datos que dan cuenta de la existencia y persistencia de la desigualdad en educación, han argumentado a favor de la necesidad de potenciar la igualdad y la justicia social en, y desde, la educación.

En el caso español, Marina Subirats $(1994,2013)$ ofrece un magnífico recorrido por la historia de la educación en España desde una perspectiva feminista, crítica, que sirve de contextualización a la problemática que se aborda en este artículo. Señala que, desde mediados del SXVIII (origen de la Escuela Moderna), se impone un modelo educativo segregado, fuertemente orientado por la religión, el catolicismo en el caso español, según el cual hombres y mujeres son creados por Dios para cumplir destinos sociales 
diferentes, por lo que la educación ha de ser también diferente para permitir, a través de una socialización de género diferenciada, que puedan cumplir esos destinos sociales diferentes por designio divino. Así, a las niñas se las orienta más hacia los rezos, las labores domésticas y una versión recortada del currículo escolar que se imparte a los niños, pues se tiene la firme convicción de que educar a las niñas podría desviarlas de su función social de madres y esposas. El siglo XIX supone un lento avance en la educación de las niñas. A finales de siglo, se empieza a defender una educación similar a niñas y niños, marcando el origen de la escuela mixta.

Esta trayectoria es común al mundo occidental, si bien, el ritmo de los cambios varía en función del país. En los países católicos de Europa, se observa un ritmo más lento. En el caso de España, la primera experiencia tiene lugar entre 1901 y 1906, cuando se establece la Escuela Moderna de Francisco Ferrer i Guàrdia en Barcelona, que va a introducir un nuevo modelo por el que apuesta con fuerza, dentro de su ideal emancipatorio: la coeducación. La escuela coeducativa ${ }^{1}$ se defiende, también, desde sectores educativos más progresistas, frente a la férrea oposición de la iglesia católica.

Sin embargo, la dictadura franquista, y el nacionalcatolicismo que la guía, suponen un período de estancamiento generalizado, de 1939 a 1970, que significa un retroceso para los derechos de las mujeres, devolviéndolas a la situación decimonónica de la segregación escolar por razón de sexo.

A partir de 1970, con la Ley General de Educación, aún bajo la dictadura franquista, se abre una nueva etapa, en que resulta fundamental la contribución de la investigación social feminista a nivel internacional en el ámbito de la educación, que supone la visibilización, con datos científicos, de las dimensiones que producen y reproducen la desigualdad de género en el sistema educativo, especialmente el currículum oculto, responsable de la socialización generizada en el ámbito escolar (Blat, 1994). Esas dimensiones son, en síntesis:

- Presencia/ausencia mujeres en sistema educativo: estudiantado, profesorado, cargos directivos.

- Incidencia del patriarcado.

- Socialización de género en el ámbito escolar.

- Uso generalizado de un lenguaje androcéntrico.

- Androcentrismo del currículum.

- Códigos de género en la cultura escolar.

- Cultura escolar androcéntrica.

- Estereotipos sexistas.

- Interacciones orientadas por las marcas de género en el seno de la comunidad educativa.

- Libros y materiales didácticos androcéntricos.

\footnotetext{
${ }^{1}$ Es importante aclarar que la terminología difiere bastante entre el inglés y el castellano. La escuela segregadora se denomina en inglés single-sex school; la escuela mix ta es coeducational school en inglés. Por tanto, cuando se habla de escuela coeducativa o coeducadora en castellano, se refiere a un modelo que apuesta explícita y sistemáticamente por la igualdad, de género, pero también en su sentido más amplio.
} 
La Ley General de Educación de 1970 es fruto de los cambios sociales, políticos y económicos que se van gestando en España desde la década anterior. Con ella se establece, por primera vez en España, el mismo currículum para niñas y niños hasta los 14 años, retomando el modelo de la escuela mixta. Sin embargo, como afirma Amparo Blat (1994), la escuela mixta es condición necesaria pero no suficiente para promover la igualdad de género. Dos fenómenos dan cuenta de la persistencia de la desigualdad de género en el ámbito escolar, como señala Subirats (2013):

- La elección generizada del tipo de estudios.

- La relación generizada entre el nivel de estudios y la forma en que se accede al mercado laboral.

Por tanto, la escuela mixta abre un espacio en el que se escolariza conjuntamente a niñas y niños, sin embargo, no elimina los mecanismos de socialización generizada en el ámbito escolar que reproducen las marcas de género en el estrecho vínculo entre formación y empleo, lo que determina las posibilidades emancipatorias de las mujeres y de la sociedad (Venegas y Lozano, 2017).

La década de 1980 va a marcar el inicio de la apuesta por el modelo coeducativo, que se va estableciendo progresivamente en algunas Comunidades Autónomas de España. La coeducación supone, pues, la concreción, en el ámbito educativo, de la apuesta institucional por la igualdad estructural. Con ello, se empiezan a incorporar valores de masculinidad y feminidad en una escuela que había sido casi exclusivamente androcéntrica, según muestra la investigación realizada desde los años 70 (Blat, 1994). Paulatinamente, sobre todo a partir de los 90, se va abriendo espacio en la escuela coeducativa también para la diversidad y la inclusión social.

Ahora bien, esta transición histórica de la segregación a la coeducación, pasando por la escuela mixta, no ha significado la erradicación del modelo segregador. Más bien, la apuesta feminista por un modelo que activamente promueva la igualdad de género en y desde la educación coexiste con el modelo diferenciado que defiende la segregación, esgrimiendo diversos argumentos, que se analizan a continuación.

Cabe hacer una distinción temporal. Por un lado, encontramos un argumento que ha prevalecido históricamente, señalado más arriba: mujeres y hombres tienen destinos sociales diferenciados, que han justificado, a su vez, esas escuelas diferenciadas. Este argumento estaría a caballo entre la fe religiosa y la fundamentación biológica: si mujeres y hombres tienen cuerpos anatómicamente diferenciados, si sus genitales tienen una función biológica (reproductiva) diferente, es porque han sido creados por Dios para desempeñar funciones sociales también diferenciadas, por lo que las instituciones encargadas de enseñarles cuáles son esas funciones sociales diferenciadas, las escuelas, habrán de serlo también (Subirats, 1994). Así pues, la diferencia biológica ha servido como justificación histórica para legitimar la desigualdad sociocultural que, cuando se materializa en prácticas institucionales concretas, da lugar a la discriminación de género.

En la actualidad, este primer argumento, a caballo entre la religión y el biodeterminismo, concluye con la existencia de diferencias en el desarrollo y la maduración del cerebro entre niños y niñas que, al estar dado genéticamente, no se puede cambiar. Los avances en la neurociencia contribuyen a reforzar este primer argumento. 
El segundo argumento, derivado del anterior, es el rendimiento escolar. Señala que existen diferencias en las actitudes y los comportamientos de los niños y las niñas, y se fundamenta en los informes educativos internacionales que muestran, desde hace años, que las chicas están obteniendo mejores resultados educativos que los chicos (Rodríguez-Martínez, 2011). La escuela segregadora se justifica mediante varias tesis tales como un mayor rendimiento escolar o la disminución del nivel de violencia. Algunas posiciones señalan, incluso, que el sistema educativo actual discrimina al estudiantado masculino. En todo caso, la consecuencia es el refuerzo del determinismo tanto biológico y como cultural. Marina Subirats (2010) pone el dedo sobre la llaga al afirmar que: "justo cuando constatamos que con la escuela mixta se ha dado un gran salto adelante en el rendimiento escolar de chicos y chicas, se inicia de nuevo el debate sobre la conveniencia de la escuela segregada" (p.145).

La ONU aprobó la Convención sobre la eliminación de todas las formas de discriminación contra la mujer en 1979. En España, todas las leyes de educación que se han aprobado en democracia han hecho suya esta Convención (Fernández-González, y González, 2015). Sin embargo, el debate entre escuela segregadora y coeducativa no se ha superado nunca. El debate más reciente se establece entre la Ley Orgánica de Educación (LOE), aprobada por un gobierno socialista en 2006, y que niega la financiación pública a centros que segregan por sexo, y la Ley Orgánica de Mejora de la Calidad Educativa (LOMCE), aprobada por un gobierno conservador del Partido Popular, y que blinda la segregación educativa por sexo (Heras y Venegas, 2016; Venegas y Heras, 2016). Ya en su Preámbulo, la LOMCE apela a la educación diferenciada en función del "talento" entre estudiantes.

Todos los estudiantes poseen talento, pero la naturaleza de este talento difiere entre ellos. En consecuencia, el sistema educativo debe contar con los mecanismos necesarios para reconocerlo y potenciarlo. El reconocimiento de esta diversidad entre alumno o alumna en sus habilidades y expectativas es el primer paso hacia el desarrollo de una estructura educativa que contemple diferentes trayectorias. Esta defensa del talento que construye trayectorias diferentes supone una vuelta al argumento biológico de la diferencia natural que ha de potenciar el sistema educativo. La situación resultante es contraria al principio de igualdad de oportunidades reconocido por la Constitución Española de 1978, en su artículo 14. Cuatro son los factores que definen el debate entre la LOE (2006) y la LOMCE (2013) sobre segregación educativa en función del sexo (Venegas y Heras, 2016):

- Económicos: financiación de centros segregadores privados/concertados, frente a importantes recortes en la educación pública en los últimos años.

- Jurídicos: blindaje de la financiación pública a la educación segregada, que el Tribunal Supremo consideraba ilegal en el marco de la loe, y que la complejidad administrativo-territorial española agrava.

- Ideológicos: la segregación escolar que introduce la lomce es entendida desde los sectores que defienden la coeducación como un retroceso reaccionario a la idea de destinos sociales diferenciados, en busca de las esencias femenina y masculina; a lo que se suma el temor a que el nivel educativo del alumnado masculino siga bajando, recrudeciendo las tesis biodeterministas. 
- Pedagógicos: la dimensión pedagógica de la reforma se asienta sobre un sustrato ideológico que defiende la libertad de elección de centro por parte de las familias, acogiéndose al artículo 27 de la Constitución.

Ahora bien, resulta crucial recordar las bases científicas de este debate, y la absoluta falta, no sólo de consenso, sino de datos empíricos unívocos sobre los que fundamentar seriamente las bondades de la segregación escolar en función del sexo:

\begin{abstract}
los defensores de este modelo aducen que la separación está extendida en países como Inglaterra o Estados Unidos. $\Upsilon$ fundamentan sus bondades en las investigaciones que sostienen que las diferencias cognitivas entre sexos hacen que aprendan mejor por separado. Sin embargo, no existe un consenso científico. La revista Science publicó a finales de 2011 el artículo La seudociencia de la escolarización por sexos, en el que se señalaba -como también ha hecho la OCDE- la inconsistencia de los trabajos que muestran mejoras académicas en las escuelas separadas. (Planelles, $2013, \mathrm{~s} / \mathrm{p})$
\end{abstract}

Efectivamente, a nivel internacional abundan los estudios cuyo objetivo ha sido comparar el nivel de logro, así como la formación emocional y social, del alumnado en la escuela diferenciada y en la mixta. Así, Woodward, Fergusson y Horwood (1999) concluyen, en el ámbito de Nueva Zelanda, que la diferenciada arroja mejores resultados entre su alumnado, si bien, ello se explica, en gran medida, por diferencias entre el alumnado anteriores a su entrada en la escuela en relación a factores familiares, sociales, comportamentales y académicos (esto es, socioestructurales). Al contrario, otros estudios concluyen que no hay datos suficientes que muestren la bondad de la escuela segregada frente a la mixta (Pahlke y Hyde, 2016; Yalcinkaya y Ulu, 2012).

Yalcinkaya y Ulu (2012) sintetizan este debate, y señalan algunos aspectos fundamentales para nuestro análisis:

Lee and Bryk (1986) found positive effects associated with SS schools for both sexes (...) Girls at SS schools did more homework and enrolled in more math courses, and boys attending SS schools enrolled in more math and science courses, than did their counterparts in CE schools. (...) Jones et al. (1972) found that students from singlesex schools are more academically oriented. Studies have also suggested that ss high schools have a more serious and studious climate which is less affected by the "rating and dating" culture that occurs in CE schools (Lee E' Bryk, 1986). In contrast, CE high schools, provide a more natural social environment to prepare adolescents to take their place in a society of men and women than do single-sex schools. (Dale, 1974, p. 13)

Así pues, el trabajo de Dale (1969, 1971, 1974, citado por Woodward y otros, 1999) es considerado pionero en mostrar que la escuela mixta es más adecuada para desarrollar las necesidades educativas y sociales de la población joven.

El tema aparentemente central del debate entre la escuela segregadora y la mixta ha sido, pues, el nivel de logro académico y cómo maximizarlo. Como se ha mostrado más arriba en este artículo, ese argumento, supuestamente científico, esconde diferentes intereses y posturas, ideológicas, políticas y religiosas, principalmente. Sin embargo, una lectura más atenta muestra, asimismo, un aspecto central en este debate, pero prácticamente obviado en España: la dimensión social, emocional, señalada por Dale ya en los 70, así como la afectivosexual, que Lee y Bryk detectaron en los 80 y denominaron the rating and dating culture. Es en este punto donde enlaza la propuesta de análisis sociológico de este artículo con el debate en torno a la igualdad en educación. 
El debate internacional gira en torno la escuela segregadora frente a la mixta. En algunos casos, se da un paso más para hablar de escuela coeducadora como la que apuesta activamente por la igualdad en y desde la educación. Establecido el análisis de este debate, en el siguiente apartado se explica el vínculo entre educación afectivosexual y coeducación.

\section{La coeducación afectivosexual}

Una vez que la investigación feminista había identificado los indicadores y las dimensiones de desigualdad en educación desde los 70, se hacía necesario emprender acciones políticas y administrativas a favor de la igualdad en este ámbito (Blat, 1994). En España, fruto de ello es, entre otras acciones, la Ley orgánica de medidas de protección integral contra la violencia de género (Ley Orgánica 1/2004) y, más concretamente, su Capítulo I dedicado a las medidas a tomar en el ámbito educativo.

No es fortuito que al año siguiente se aprobara en Andalucía el I Plan de Igualdad entre Hombres y Mujeres en Educación (Consejería de Educación, 2005), en torno al cual se va a desarrollar la Colección Plan de Igualdad² para canalizar mejor el Plan de Igualdad, de entre la que cabe destacar la Guía de Buenas Prácticas orientada a dar una formación básica al profesorado (Consejería de Educación, 2006). Asimismo, en 2016 se aprueba el II Plan Estratégico de Igualdad en Educación³ (Consejería de Educación, 2016). De hecho, ya desde la década de 1990, la Junta de Andalucía venía apostando abiertamente por la coeducación (Consejería de Educación y Ciencia, 1993a, 1993b), en estrecha colaboración con el Instituto Andaluz de la Mujer. En el I Plan se plantean algunos retos destacados para fomentar la igualdad entre mujeres y hombres en el ámbito educativo, de entre los que cabe destacar uno en el marco del objetivo que nos ocupa en este artículo, a saber: la introducción de una asignatura optativa en el segundo ciclo de Educación Secundaria Obligatoria, Cambios sociales y nuevas relaciones de género, que según el balance realizado del I Plan ha sido impartida en el 50\% de los centros andaluces.

Sin embargo, como muestran las investigaciones internacionales, España sigue careciendo de políticas educativas que canalicen la educación afectivosexual en el sistema educativo formal. Así lo señalan en su artículo Parker, Wellings y Lazarus (2009) the Spanish government has no explicit sexual and reproductive health policy, and there are no set minimum standards for the provision of sexuality education. Nor is the subject mandatory in schools (p. 239). Al año siguiente a la publicación de este artículo, el gobierno socialista aprueba la Ley Orgánica 2/2010, de 3 de marzo, de salud sexual y reproductiva y de la interrupción voluntaria del embarazo, cuyo Capítulo III está dedicado a las Medidas en el ámbito educativo, de manera que el Artículo 9 se refiere a la incorporación de la formación en salud sexual y reproductiva al sistema educativo, y el Artículo 10 a actividades formativas. Nótese que no se trata de la mal llamada "Ley del Aborto", sino de una ley que, por primera vez en España, incorpora los derechos de salud reproductiva y sexual, reconocidos como derechos humanos, al tiempo que traslada al ámbito educativo esta relevante cuestión, afirmando de manera pionera la necesidad de

2 Véase en: http://www.juntadeandalucia.es/educacion/webportal/web/portal-de-igualdad/coleccion-plan-de-igualdad 3 Sin duda, los cambios terminológicos del II Plan no son baladíes, ni fortuitos, pero ese análisis queda fuera de los objetivos de ese artículo, y merece uno aparte. 
incorporar la educación, no sexual, sino afectivosexual, al currículum educativo formal. No se ha visibilizado lo suficiente la importancia de esta dimensión de la ley, porque la visibilidad mediática y el debate social se han centrado en su parte más polémica: los plazos legales para abortar y la posibilidad de las menores de abortar sin consentimiento de, ni necesidad de informar a, sus padres o tutores legales. Sin embargo, se trataba de una ley de gran importancia para la educación y la igualdad que, con la llegada del Partido Popular al gobierno un año más tarde, se ha quedado sin implementar.

Otro aspecto relevante en torno a las cuestiones que plantea este texto es, pues, la diferencia entre educación sexual y educación afectivosexual, y la posición que la opción por cada una de ellas implica en términos educativos y sociales (Venegas, 2011). La mayor parte de documentos disponibles en España sobre la educación para la sexualidad desde finales de los 80 aborda esta cuestión en términos de educación sexual. Sólo algunos de ellos incluyen la dimensión afectiva, que se entiende aquí como relacional. Este hecho explica la ausencia de la dimensión afectivo-relacional en los programas educativos, dando lugar a una paradoja: por un lado, la afectividad emerge como área fundamental en educación sexual; por otro, sin embargo, la afectividad está más implícita que formulada explícitamente en esos programas, se intuye como dimensión que interacciona inevitablemente con la sexualidad, especialmente en la adolescencia, pero no se formula en detalle, como se hace con la sexualidad, algo que, por otro lado, no es de extrañar si reparamos en el hecho de que tampoco a nivel de producción teórica y empírica la afectividad ha ocupado un espacio amplio como estructura de relaciones sociales (Venegas, 2013a).

La educación sexual estaría, pues, más enfocada a la salud y menos a educar las relaciones adolescentes en ese espacio que son la sexualidad y las relaciones sexuales en el marco de la coeducación. Es decir, la educación sexual tiene una orientación más biológica, centrada en la anatomía, los genitales y la función reproductora del ser humano, por lo que pone especial énfasis también en la prevención, tanto de embarazos no deseados -adolescentes-, como de infecciones o enfermedades de transmisión sexual. Sin embargo, desde la mirada sociológica que se adopta en este artículo, el interés radica más en la dimensión social, relacional, presente en las relaciones afectivosexuales, donde la afectividad remite en sí misma a una forma de relación, afectada positivamente o negativamente. La afectividad en la sexualidad, desde esta mirada, no contiene un interés moralizante, sino educativo. Al entender la sexualidad como forma de relación social, conlleva implícita la afectividad, de una persona hacia sí misma y hacia otras personas. La educación afectivosexual remite, pues, a la política de las relaciones afectivosexuales, entendiendo por política la presencia del poder y la desigualdad consecuente a ese poder en toda relación social. Para incidir directamente en esa desigualdad, que en este caso implica la perspectiva de género, es para lo que resulta necesario contextualizar la educación afectivosexual en el marco de la coeducación, como modelo que pretende promover la igualdad sexual y de género:

Gender is a structuring principle in all societies. All social relations, including gender relations, are structural relations mediated by power (Connell 1987; Foucault 1989). Because of this, I will use the term the politics of sexuality and relationships (in Spanish, politica afectivosexual) to refer to the merging of gender, sexuality, affection and the body, wherein politics means the principles relating to or inherent in a sphere or activity, especially when concerned with power and status (Soanes y Stevenson 2008, 1110). Hence, the politics of sexuality and relationships 
is concerned with the power that mediates gender, sexual, affective and bodily

relations, and their multiple links. (Venegas, 2013b, pp. 573-574)

De ahí que se hable aquí de coeducación afectivosexual como fenómeno sociológico. El modelo coeducativo de educación afectivosexual, democrático y holístico, se caracteriza por (Venegas, 2011, 2013c):

- un enfoque constructivista y de género para promover cambio social hacia la igualdad sexual y de género,

- un espíritu pedagógico y una visión integral de la persona,

- enfatizando el autoconocimiento de nuestro cuerpo y nuestra sexualidad, para posibilitar el empoderamiento de todas las personas,

- definiendo sexualidad como dimensión fundamental del ser humano, entendida no sólo para la reproducción biológico sino, muy importante, como forma de comunicación, relación, afectividad y placer,

- en que las personas son consideradas como sujetos agentes de su cuerpo y de su vida afectivosexual,

- desarrollando la ciudadanía también en lo afectivosexual,

- este enfoque implica un giro epistemológico, de la educación a la política afectivosexual,

- así como un giro metodológico, del individualismo metodológico al enfoque centrado en el componente relacional y la naturaleza socioestructural de la dimensión afectivosexual de las personas.

Por tanto, junto a la Ley contra la violencia de género de 2004, que introduce el marco legislativo para la coeducación; la Ley de salud reproductiva y sexual e interrupción voluntaria del embarazo de 2010, que hubiera materializado la educación afectivosexual en el sistema educativo formal; y el desarrollo de acciones y medidas para favorecer la coeducación, como en el caso andaluz donde, además, hay que considerar elementos como la optativa de Educación Secundaria "Cambios sociales y nuevas relaciones de género", con un capítulo dedicado a las "Relaciones y sentimientos". Estos hitos abren un espacio para reflexionar sobre esa importante relación sociológica entre coeducación (frente a segregación educativa por sexo), educación afectivosexual, igualdad y justicia social desde el enfoque feminista del cambio social. Una relación que, de alguna manera, quedara apuntada ya desde los años 70 y 80 en investigaciones como las de Dale, o Lee y Bryk. Pero esta imagen no está completa si, junto al debate entre modelos educativos y su impacto en la formación de los sujetos (logro educativo, desarrollo emocional y social), no se ilustra con investigaciones sociológicas que permitan dar cuenta de esa realidad social, como se muestra en la siguiente sección.

\section{La necesidad de coeducar las relaciones afectivosexuales para promover la igualdad sexual y de género y la justicia social}

Una vez definidos los parámetros conceptuales y teóricos de la coeducación afectivosexual, a continuación, mediante análisis de fuentes secundarias, en concreto, 
tres estudios del Centro de Investigaciones Sociológicas (CIS, 2008a, 2008b, 2010), se pasa a analizar actitudes y prácticas afectivosexuales en España, con especial atención a la adolescencia-juventud (CIS, 2010), para fundamentar, sobre dichos datos, la necesidad de apostar seria y firmemente por coeducar las relaciones afectivosexuales para promover la igualdad sexual y de género y la justicia social.

Para hacer un análisis de los datos disponibles sobre este tema, se seguirán las tres únicas encuestas realizadas por el CIS que, si bien datan de hace algunos años, son las únicas disponibles en España sobre el fenómeno que nos ocupa:

- E1 (CIS, 2008a): sobre "Actitudes y prácticas sexuales" (desglosada por sexo). Realizada el 14 de enero de 2008, a un total de 1503 personas, de ámbito nacional, siendo el universo población residente en España, de ambos sexos, y de 18 años o más, por encargo del Ministerio de Sanidad y Consumo.

- E2 (CIS, 2008b): se trata de la "Encuesta nacional sobre salud sexual" (desglosada por sexo). Realizada el 17 de noviembre de 2008, a un total de 9850 personas, de ámbito nacional, siendo el universo población residente en España, de ambos sexos, y de 16 años o más, por encargo del Ministerio de Sanidad y Consumo.

- E3 (CIS, 2010): sobre "Actitudes de la juventud ante la diversidad sexual" (desglosada por sexo). Realiza el 23 de noviembre de 2010, a un total de 1411 personas, de ámbito nacional, siendo el universo población residente en España, de ambos sexos, y de 15 a 29 años, por encargo del Instituto de la Juventud.

Para no hacer una presentación farragosa en exceso aquí, con un sinfín de tablas elaboradas por el CIS, y puesto que los tres estudios a los que se refiere este análisis son de acceso gratuito y pueden consultarse en su web, lo que se presentará a continuación es una discusión, en síntesis, de algunos aspectos relevantes para el objetivo de este artículo.

El ideal de la pareja estable. Un $78 \%$ de las personas encuestadas en la E1 tiene pareja estable en el momento de hacer la entrevista. De esas personas, un 30,7\% lleva más de 30 años con esa pareja, siendo éste dato el mayor porcentaje de todos los registrados en esa pregunta.

La edad de la primera relación sexual. Un 6,6\% de personas encuestadas en la E1 afirma haber tenido relaciones sexuales antes de los 15 años, siendo un $56,2 \%$ hasta los 18 años (32\%, entre 17-18 años). El 7,7\% reconoce haberla tenido con más de 24 años. Ahora bien, desde la postura que se defiende en este trabajo, la duda inmediata que surge es: ¿qué se entiende por relación sexual? Desde el modelo de coeducación afectivosexual expuesto más arriba, relación sexual es toda forma de relación que implica la sexualidad, desde un beso, una caricia, un abrazo, etc. Sin embargo, en la encuesta del CIS y, con seguridad, en el imaginario de las personas que han respondido a la misma, primera relación sexual será equivalente a primera relación coital. Al identificar la sexualidad con el coito, se reduce a la genitalidad y la penetración, exclusivamente, lo que priva a la sexualidad de esa visión integral que se señalaba antes. No es, pues, una pregunta formulada correctamente.

La primera relación sexual. Esta debilidad se corrige en la E2, donde se pregunta por la primera relación sexual, señalando en el enunciado de la pregunta (P10) que: "En esta investigación se considera relación sexual al conjunto de prácticas que realizan dos o 
más personas con la finalidad de obtener placer sexual, que no tiene por qué incluir el coito, ni concluir con el orgasmo", una definición más acorde con el modelo que defiende este artículo. Besos y caricias es la respuesta más señalada, ligeramente mayor entre las mujeres $(89,1 \%$ de las mujeres y $87,5 \%$ de los hombres); seguida de sexo vaginal $(48,9 \%$ de las mujeres reconocen que se les ha realizado sexo vaginal; $49,3 \%$ de los hombres reconoce haber realizado sexo vaginal a otra persona); la tercera opción más señalada es la masturbación mutua (señalada por el 27\% de los hombres y el 17,3\% de las mujeres).

La E2 pregunta por la persona con la que se mantiene la primera relación sexual, siendo la pareja estable en aquel momento para el $85,9 \%$ de las mujeres y para la mitad de los hombres, el 46,3\%, lo que muestra el peso que aún tiene la pareja estable como marco legítimo para la sexualidad de las mujeres. Así, para el 36,1\% de los hombres fue en una relación ocasional o esporádica, mientras que esta opción la señala sólo el 10,8\% de las mujeres.

El sentido de la sexualidad. La E2 pregunta también por el significado de la sexualidad para las personas encuestadas, siendo la respuesta claramente más señalada "La sexualidad es principalmente un medio para buscar comunicación, placer, afecto, ternura e intimidad", una respuesta en línea con el modelo democrático antes definido en este artículo, siendo un poco mayor el porcentaje de mujeres, $79,2 \%$, que el de hombres, $73,8 \%$. También es de destacar que para el 16,3\% de los hombres (el doble que entre las mujeres) "La sexualidad es un medio para buscar placer y satisfacer el deseo", mientras que es mayor el porcentaje de mujeres $(9,3 \%)$ que de hombres $(6,7 \%)$ que consideran que "La sexualidad es principalmente un medio para tener hijos/as". El sentido de la sexualidad es retomado por la E3, con las mismas categorías de respuesta. En este caso, "La sexualidad es principalmente un medio para buscar comunicación, placer, afecto, ternura e intimidad" es la respuesta del $74,1 \%$ de personas encuestadas (recuérdese que se trata de mujeres y hombres de 15-29 años); "La sexualidad es un medio para buscar placer y satisfacer el deseo" es la respuesta del 16,7\% y sólo para el 5,5\% "La sexualidad es principalmente un medio para tener hijos/as". La E3 no ofrece los datos desglosados por sexo, pero nos permite ver que para la gente más gente la sexualidad como reproducción es menos importante, y lo es más la sexualidad como placer, aunque un poco menos como comunicación, afecto e intimidad.

Los temas esperados en educación afectivosexual. La E2 pregunta por lo que les hubiera gustado haber recibido más información. Para los hombres, por este orden de prioridad y con porcentajes sobre 20\% de respuesta: Prevención de infecciones de transmisión sexual, VIH-SIDA; Métodos anticonceptivos; Amor y relaciones sentimentales; Problemas en las relaciones sexuales; Maneras de obtener y dar placer. Para las mujeres: Métodos anticonceptivos; Prevención de infecciones de transmisión sexual, VIH-SIDA; Amor y relaciones sentimentales; Problemas en las relaciones sexuales; Reproducción; Maneras de obtener y dar placer.

Conciencia y prevención. La E 1 pregunta también por la protección en relaciones sexuales esporádicas u ocasionales, tanto de embarazos, como de contagios, los porcentajes son algo más de la mitad reconoce que "Sí, siempre", pero en torno a un tercio reconoce que "No nuca", siendo algo mayor el porcentaje de quienes no se protegen nunca de posibles contagios. Tras la grave crisis del sida de los 90, y la fuerte concienciación posterior, vivimos un momento de relajación en este aspecto, algo que debe preocuparnos. 
Educación. La E2 pregunta si se recibió algún tipo de educación sexual (claro que no aclara qué se entienda por ello). El 67,6\% reconoce no haber recibido educación sexual nunca. Entre el 32,2\% que sí recibió, un 14,2\% fue antes de los 12 años y un 17,1\% con más de 15 años. La mayor frecuencia está en los 14 años (20,9\%). Sin embargo, dato muy relevante es el 93,9\% que considera que debería darse educación sexual en los colegios, siendo de este grupo el 22,2\% quienes piensan que tendría que ser antes de los 10 años, y el 15,1\% con más de 13 años, lo que apunta a una demanda social de abordar la educación sexual en los colegios antes de la adolescencia, un dato especialmente importante en el marco de la reivindicación que se hace en este artículo.

Agentes educativos. Al preguntar la E2 por la persona a través de la que les hubiera gustado recibir información acerca de la sexualidad, las mujeres encuestadas señalan mayoritariamente a la madre (58,9\%), seguida del profesorado en el colegio (13,8\%), siendo la tercera opción el personal sanitario (9\%), y el padre una opción muy minoritaria (para sólo el 2,4\% de las mujeres encuestadas) y difuminada entre el resto de respuestas minoritarias. Entre los hombres encuestados se destaca en primer lugar al padre $(35,5 \%)$, seguido del profesorado del colegio $(20,3 \%)$, siendo la madre la tercera opción (para el 12,7\%). Como siempre que es posible aplicar el enfoque de género, se observan las marcas de género en la socialización sexual de los sujetos encuestados. También la E3 recoge esta cuestión, al preguntar por la principal fuente de información en temas de sexualidad. La primera opción son amistades y compañeros (24,4\%); seguida de profesorado o personal del instituto $(24,2 \%)$, cabe señalar en este sentido que la muestra de la encuesta tiene una edad comprendida entre 15-29 años, por lo que el ámbito educativo constituye un espacio de referencia de especial relevancia, que no existe en las otras dos encuestas.

Pagar, abusar, dos marcas diferenciales de género. Esas marcas de género se encuentran también en las preguntas relacionadas con el pago por mantener relaciones sexuales (casi un tercio de hombres, 32,1\%, ha pagado al menos una vez por tener relaciones sexuales frente al $0,3 \%$ de mujeres que lo ha hecho; al 3,1\% de hombres encuestados le han pagado al menos una vez por mantener relaciones sexuales, frente al $0,6 \%$ de mujeres que dice haberlo hecho), así como por haber sufrido abusos o relaciones sexuales contra su voluntad $(1,7 \%$ de hombres dice haber sufrido abusos al menos una vez, frente al $5,2 \%$ de mujeres que dice haberlos sufrido; $3,5 \%$ de hombre dice haber mantenido relaciones sexuales contra su voluntad al menos una vez, frente a $7,4 \%$ de las mujeres encuestadas). Se trata de fenómenos marcadamente importantes desde una óptica feminista de la sexualidad y que nos dan cuenta de la importancia de apostar firmemente por la coeducación afectivosexual para la igualdad y la justicia social.

El ámbito educativo, gran ausente. La E2 se interesa también por la persona a la que se acude en caso de necesitar buscar ayuda ante una preocupación por su vida sexual. La primera opción señala son profesionales (para el 58,5\% de los hombres encuestados, y el 60,3\% de las mujeres); la segunda opción son los amigos para 25,6\% de los hombres, y las amigas para el 20,3\% de las mujeres (nótese que los hombres hablan de sus amigos, en masculino, y las mujeres de sus amigas, en femenino); la tercera opción es la pareja o cónyuge (para el 9,4\% de los hombres y el $12 \%$ de las mujeres encuestadas); la cuarta opción es la madre (para el 7,3\% de hombres y el 11,3\% de mujeres). El resto de opciones está más disperso, pero el padre no ocupa un lugar destacado entre ellas, lo que señala a la madre como agente educativo y socializador más destacado que el padre también en torno a la sexualidad, lo que prolonga el papel de las madres como cuidadoras. El 
profesorado del colegio, que está entre las opciones de respuesta a esta pregunta, es señalado sólo por el 0,2\% de los hombres encuestados, siendo $0 \%$ entre las mujeres, lo que muestra la clara ausencia que supone el ámbito educativo como agente de educación afectivosexual en el imaginario colectivo en España. También la E3 pregunta por la frecuencia con la que se hablaba de sexualidad en el centro de estudios: el $48,7 \%$ señala que "con poca frecuencia", y el 32,6\% "con bastante frecuencia", siendo un 11,8\% las personas que señalan "con ninguna frecuencia, nunca".

La familia, otra gran ausente. La E3 se interesa por la frecuencia con que se hablaba de sexualidad en las conversaciones familiares durante la infancia y la adolescencia. El $48,7 \%$ de personas encuestadas reconoce que se hablaba "con poca frecuencia", y el 30,8\% "con ninguna frecuencia, nunca"; el 16,7\% señala "con bastante frecuencia" y sólo el $2,8 \%$ señala "con mucha frecuencia". O sea, que hablar de sexualidad en el hogar, con la familia, es prácticamente inexistente en España.

\section{Concluyendo}

Este artículo parte del origen de la educación moderna para llegar al momento actual. En este recorrido, el artículo ha querido reparar en algunos hitos destacados en la relación entre la escuela y la igualdad de género, para analizar cómo partimos de la segregación educativa en función del sexo y, tras importantes esfuerzos por la igualdad de género, transitamos hacia la escuela mixta, primero, y la coeducativa, más tarde, para volver a un momento en que las posiciones políticas más conservadoras apuestan y protegen la escuela segregadora de nuevo, lo que nos hace retroceder a la situación decimonónica desigualitaria.

Frente al modelo diferenciado que segrega, la coeducación ofrece un modelo que apuesta por la igualdad sexual y de género y que, paulatinamente, ha ido incorporando elementos para educar también las relaciones afectivosexuales. Esta cuestión ha ocupado la última parte de este artículo, donde se ha descrito un modelo democrático de coeducación afectivosexual para la igualdad y la justicia social, para pasar a analizar algunos datos relevantes sobre la política afectivosexual en España según los tres estudios sociológicos disponibles realizados por el CIS (2008a, 2008b, 2010).

Entre estos aspectos, cabe destacar aquí, a modo de conclusión, los siguientes. A pesar de los cambios en los modelos de relación, amor y sexualidad (Venegas y Lozano, 2017), la pareja y, en concreto, estable sigue siendo el ideal de modelo de relación. Sin tener muy claro qué se entiende por primera relación sexual, el grueso de la sociedad española parece tenerla a los 18 años o antes. En cuanto al sentido de la sexualidad, y aunque no se pregunta lo que se entiende por sexualidad, por lo que partimos de un vacío en ese sentido, la mayoría de la sociedad española parece entender la sexualidad como medio para buscar comunicación, placer, afecto, ternura e intimidad, lo que supone un cambio muy importante con respecto al sentido tradicional y normativo de la reproducción. Prevención de ETS y embarazos no deseados siguen siendo los dos temas más esperados por la sociedad cuando se trata de educación sexual, quedando en un segundo plano temas más propios de la educación afectivosexuales como amor y relaciones. El placer quedaría en tercera posición. Se observa una preocupante falta de conciencia sobre la importancia de protegerse en las relaciones (coitales) frente a embarazos y contagios. La educación sexual es una gran ausente en la escuela, como son grandes ausentes familia y 
escuela en la educación (afectivo)sexual. Familia y escuela son los dos agentes de socialización por excelencia (Venegas, 2017). Sin embargo, a tenor de los datos del CIS, son los grandes ausentes de la socialización afectivosexuales en España, lo que significa, pues, que otros agentes de socialización están ocupando ese lugar, con la importancia que ello supone para construir relaciones afectivosexuales basadas en la igualdad y la justicia social. Otro de los aspectos más destacado al analizar los datos del CIS es el diferencial de género existente en todo lo relacionado con la sexualidad y las relaciones.

De este modo, cabe concluir diciendo que los datos del CIS revelan algunos aspectos especialmente relevantes sobre los que es posible fundamentar la necesidad de apostar por la coeducación afectivosexual como espacio educativo, socializador, imprescindible para construir relaciones afectivosexuales democráticas, basadas en la igualdad sexual y de género, el respeto, la diversidad y la justicia social, esto es, apostar por la coeducación afectivosexual en el marco de la educación para la ciudadanía democrática y los derechos humanos (Venegas, 2013c).

\section{Referencias}

Araújo, H. C. (2000). Pioneiras na educação: As professoras primárias, percursos, experiências e subjectividades, 1870-1933. Lisboa: Instituto de Inovação Educacional.

Arnot, M. y Weiler, K. (1993). Feminism and social justice in education: International perspectives. Londres: Falmer Press.

Blat, A. (1994). Informe sobre la igualdad de oportunidades educativas entre los sexos. Revista Iberoamericana de Educación, 6, 23-145.

Centro de Investigaciones Sociológicas. (2008a). Actitudes y prácticas sexuales (2738). Recuperado de http://www.cis.es/cis/opencm/ES/1_encuestas/estudios/ver.jsp?estudio=9882

Centro de Investigaciones Sociológicas. (2008b). Encuesta nacional de salud sexual (2780). Recuperado de http://www.cis.es/cis/opencm/ES/1_encuestas/estudios/ver.jsp?estudio=9702\&cuestion ario $=11296 \&$ muestra $=17045$

Centro de Investigaciones Sociológicas. (2010). Actitudes de la juventud ante la diversidad sexual (2854) Recuperado

de http://www.cis.es/cis/opencm/ES/1_encuestas/estudios/ver.jsp?estudio=11984

Consejería de Educación y Ciencia. (1993a). Programa de coeducación. Sevilla: Junta de Andalucía.

Consejería de Educación y Ciencia. (1993b). Campaña de sensibilización en coeducación. Sevilla: Junta de Andalucía.

Consejería de Educación. (2005). I Plan de igualdad entre mujeres y hombres en educación. Sevilla: Junta de Andalucía.

Consejería de Educación. (2006). Guía de buenas prácticas para promover la igualdad entre mujeres y hombres en educación. Sevilla: Junta de Andalucía.

Duru-Bellat, M. (1990). Lécole des filles: Quelle formation pour quels rôles sociaux? París: LHarmattan.

Fernández-González, N. y González, N. (2015). La LOMCE a la luz de la CEDAW. Un análisis de la coeducación en la última reforma educativa. Journal of Supranational Policies of Education, 3, 242-263. 
Heras, P. y Venegas, M. (2016). Educación afectivosexual y política educativa: Un análisis comparado entre la LOE y la LOMCE. En J. Aznar e I. Belmonte (Eds.), Las políticas de igualdad: Una visión calidoscópica tras cinco años de experiencia (pp. 56-75). Elche: Universidad Miguel Hernández.

Lee, V. E. y Bryk, A. S. (1986). Effects of single-sex secondary schools on student achievement and attitudes. Journal of Educational Psychology, 78(5), 381-395. https://doi.org/10.1037/0022-0663.78.5.381

Pahlke, E. y Hyde, J. (2016). The debate over single-sex schooling. Child Development Perspectives, IO(2), 81-86. https://doi.org/10.1111/cdep.12167

Parker, R., Wellings, K. y Lazarus, J. V. (2009). Sexuality education in Europe: An overview of current policies. Sex Education: Sexuality, Society and Learning 9(3), 227-242. https://doi.org/10.1080/14681810903059060

Planelles, M. (12 de febrero de 2013). Andalucía niega el concierto a los 12 colegios que separan por sexo. $E l$ País. Recuperado de http://ccaa.elpais.com/ccaa/2013/02/12/andalucia/1360665805_226740.html

Rodríguez-Martínez, C. (2011). Rendimientos escolares, género y segregación escolar. En M. Jiménez y F. J. Del Pozo (Eds.), Propuestas didácticas de educación para la igualdad (s/p). Granada: Natívola.

Subirats, M. (1994). Conquistar la igualdad: La coeducación hoy. Revista Iberoamericana de Educación, 6(3), 49-78.

Subirats, M. (2010). ¿Coeducación o escuela segregada? Un viejo y persistente debate. Revista de la Asociación de Sociología de la Educación, 3(1), 143-158.

Subirats, M. (2013). Género y educación. En C. Díaz y S. Dema (Eds.), Sociología y género (pp. 201-252). Madrid: Biblioteca Universitaria de Editorial Tecnos.

Subirats, M. y Brullet, C. (2002). Rosa y azul: La transmisión de los géneros en la escuela mixta. En A. González (Ed.), Mujer y educación: Educar en la igualdad, educar desde la diferencia (pp. 133-168). Barcelona: Graó.

Venegas, M. (2011). El modelo actual de educación afectivosexual en España. El caso de Andalucía. Revista Iberoamericana de Educación, 55(3), 1-10.

Venegas, M. (2013a). Amor, sexualidad y adolescencia. Sociología de las relaciones afectivosexuales. Granada: Comares.

Venegas, M. (2013b). Sex and relationships education and gender equality: Recent experiences from Andalusia (Spain). Sex Education: Sexuality, Society and Learning, 13(5), 573-584,

Venegas, M. (2013c). La educación afectivosexual en el marco de la educación para la ciudadanía democrática y los derechos humanos. Revista de la Asociación de Sociología de la Educación, $6(3), 408-425$.

Venegas, M. (2017). Devenir sujeto. Una aproximación sociológica. Convergencia. Revista de Ciencias Sociales, 73(1), 13-36.

Venegas, M. y Heras, P. (2016). Financiar la segregación educativa: Un debate sobre la LOMCE desde una perspectiva crítica de género. Revista Educación, Política y Sociedad, 1(2), 73-99.

Venegas, M. y Lozano, A. (2017). Educación, empleo y transformación de la estructura familiar: El papel de la igualdad. En D. Becerril y M. Venegas (Eds.), La custodia compartida en España (pp. 25-43). Madrid: Dykinson. 
Woodward, L. J., Fergusson, D. M. L. y Horwood, J. (1999). Effects of single-sex and coeducational secondary schooling on children's academic achievement. Australian Journal of Education, 43(2), 142-156. https://doi.org/10.1177/000494419904300204

Yalcinkaya, M. T. y Ulu, A. (2012). Differences between single-sex schools and co-education $\begin{array}{llll}\text { schools. Procedia-Social and Behavioral Sciences, } 46, & 13-16 .\end{array}$ https://doi.org/10.1016/j.sbspro.2012.05.058

\section{Breve CV de la autora}

\section{Mar Venegas}

Licenciada en Sociología (2002), Experta en Género e Igualdad de Oportunidades (2004) y Doctora en Sociología con Mención de Doctorado Europeo (2009) por la Universidad de Granada. Es profesora del Dpto. de Sociología de esta Universidad y Subdirectora desde diciembre de 2014. Es Vicedirectora de la Revista de la Asociación de Sociología de la Educación (RASE) / ISSN: 1988-7302 desde julio de 2016. Es Codirectora del Curso Certificado Oficial de Formación Pedagógica y Didáctica Equivalente de Universidad Internacional de Andalucía. Ha sido secretaria de la Asociación de Sociología de la Educación de España entre los años 2014-2016. Su investigación y su docencia se desarrollan en el ámbito de la Sociología de la Educación y los Estudios de Género y Sexualidad, siendo dos sus líneas de investigación principales: Educación/Política afectivosexual y Profesorado. ORCID ID: 0000-0001-7500-8005. Email: mariter@ugr.es 